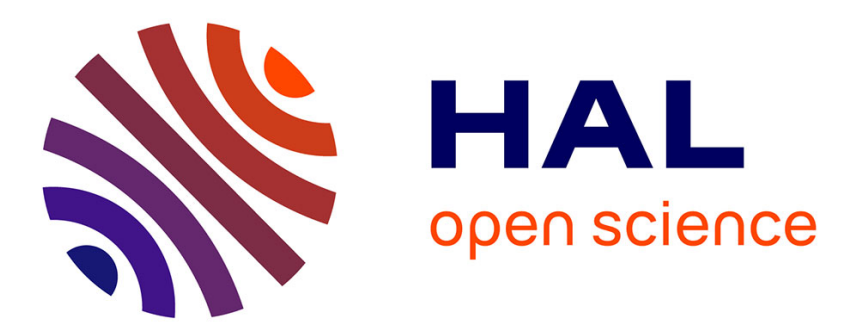

\title{
Transmission gaps and sharp resonant states in the electronic transport through a simple mesoscopic device
}

Housni Al-Wahsh, El Houssaine El Boudouti, Bahram Djafari-Rouhani, L. Dobrzynski, Abdellatif Akjouj

\section{- To cite this version:}

Housni Al-Wahsh, El Houssaine El Boudouti, Bahram Djafari-Rouhani, L. Dobrzynski, Abdellatif Akjouj. Transmission gaps and sharp resonant states in the electronic transport through a simple mesoscopic device. Physical Review B: Condensed Matter and Materials Physics (1998-2015), 2007, 75 (12), pp.125313. 10.1103/PhysRevB.75.125313 . hal-00283159

\section{HAL Id: hal-00283159 \\ https://hal.science/hal-00283159}

Submitted on 16 Aug 2021

HAL is a multi-disciplinary open access archive for the deposit and dissemination of scientific research documents, whether they are published or not. The documents may come from teaching and research institutions in France or abroad, or from public or private research centers.
L'archive ouverte pluridisciplinaire HAL, est destinée au dépôt et à la diffusion de documents scientifiques de niveau recherche, publiés ou non, émanant des établissements d'enseignement et de recherche français ou étrangers, des laboratoires publics ou privés. 


\title{
Transmission gaps and sharp resonant states in the electronic transport through a simple mesoscopic device
}

\author{
H. Al-Wahsh, ${ }^{1,2}$ E. H. El Boudouti, ${ }^{1,3}$ B. Djafari-Rouhani, ${ }^{1}$ A. Akjouj ${ }^{1, *}$ and L. Dobrzynski ${ }^{1}$ \\ ${ }^{1}$ Institut d'Electronique, de Microélectronique et de Nanotechnologie (IEMN), UMR CNRS 8520, UFR de Physique, \\ Université des Sciences et Technologies de Lille, 59655 Villeneuve d'Ascq Cédex, France \\ ${ }^{2}$ Faculty of Engineering, Benha University, 11241 Cairo, Egypt \\ ${ }^{3}$ Laboratoire de Dynamique et d'optique des matériaux, Département de Physique, Faculté des Sciences, \\ Université Mohamed I, 60000 Oujda, Morocco
}

(Received 8 June 2006; revised manuscript received 4 August 2006; published 16 March 2007)

\begin{abstract}
A simple electronic circuit consisting of a single symmetric or asymmetric loop with dangling resonators is designed to obtain possibly large stop bands (where the propagation of electrons is forbidden). Contrary to all known systems of this kind, a spectral transmission gap of nonzero width occurs here even with a single loop. This is obtained by combining appropriately the zeros of transmission of the loop and of the dangling resonators. Sharp resonant electronic states inside the gaps can be achieved without introducing any defects in the structure. This results from an internal resonance of the structure when such a resonance is situated in the vicinity of a zero of transmission or squeezed between two zeros of transmission, the so-called Fano resonances. A general expression for the transmission coefficient is given for various systems of this kind within the framework of the interface response theory. The amplitude and the phase of the transmission are discussed as a function of the wave vector or energy and it is shown that the width of the stop bands is very sensitive to the number of grafted resonators, while the magnitude of the resonant states in the transmission coefficient is very sensitive to the lengths of the different arms constituting the loop and the dangling resonators. These structures may have potential applications in microelectronic devices.
\end{abstract}

DOI: 10.1103/PhysRevB.75.125313

PACS number(s): 73.21.-b, 73.63.-b, 73.20.At

\section{INTRODUCTION}

Electronic transport in low-dimensional systems, i.e., mesoscopic structures with a dimensionality less than 3 has attracted enthusiastic attention in the past few years. ${ }^{1}$ This is related to the impressive development of nanoscience, which places the detailed understanding of quantum transport in mesoscopic systems. A rich variety of quantum devices and structures, including simple metallic wires, as well as complex molecules, where electrons are driven by some external force, are the subject of experimental and theoretical investigations. ${ }^{2-7}$ Progress in nanofabrication ${ }^{8}$ has achieved devices of the size of the order of the various coherence lengths of the conduction electrons. Therefore, different quantum mechanical effects such as Fano resonances, ${ }^{9,10}$ Aharonov-Bohm oscillations, ${ }^{11,12}$ persistent currents, ${ }^{13}$ and current magnification ${ }^{14}$ are observed and analyzed. These devices can be used in the probe of phase coherency for electrons in transport ${ }^{15}$ and the design of mesoscopic spin filters. ${ }^{16}$

The motivation behind the work presented in this paper is to introduce a design of a simple electronic circuit consisting of a loop with dangling resonators connected to two leads (Fig. 1). This system has the same configuration as that of the commonly studied Aharonov-Bohm system with one $e^{9,10}$ or two ${ }^{17}$ coupled quantum dots on one arm (the other being considered as a reference) or with one quantum dot on both arms. ${ }^{18}$ Mainly, the subject of these studies was to use this interferometric system to show the conditions for the existence and the collapse of Fano resonances as function of the applied current voltage and magnetic flux. These studies are also related to the investigation of the electronic states of quantum dots incorporated into the arms $\mathrm{s}^{9,10}$ as well as to the understanding ${ }^{19}$ of the transmission phase jumps by $\pi$ between two adjacent resonances in relation with the experiments of Yacoby et al. ${ }^{12}$ The aim of this work is different from those cited above. Indeed, we show that a simple loop with dangling resonators may produce a large stop band in the electronic band spectrum as well as sharp resonances inside the gaps, which have been established through an analysis of the transmission function (amplitude and phase) obtained within the framework of the Green's function method. ${ }^{20}$ We show that this simple structure may exhibit large gaps without repeating it periodically, as it is usually the case with such structures..$^{21}$ also, one can obtain strong and sharp resonances by tailoring the lengths of the different wires constituting this structure. This could be of potential interest for developing nanocircuits that may play the role of reflectors or filters for electrons.

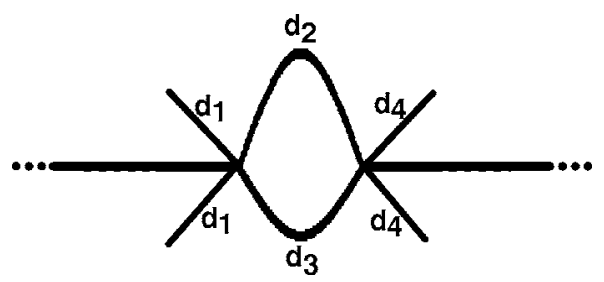

FIG. 1. Schematic illustration of the one-dimensional loop structure with dangling resonators on both sides; the whole structure is inserted between two semi-infinite leads. The lengths of the two arms of the loop are $d_{2}$ and $d_{3}$, whereas the lengths of the dangling resonators are $d_{1}$ and $d_{4}$ and their numbers are $N$ and $N^{\prime}$, respectively (here, $N=N^{\prime}=2$ ). 
In previous publications, ${ }^{22,23}$ we proposed a model of onedimensional (1D) monomode waveguide exhibiting passbands separated by large forbidden bands. The geometry of the model presented in Ref. 22 [called a comblike structure $(\mathrm{CLS})]$ is composed of an infinite 1D monomode waveguide (the backbone) along which $N^{\prime}$ dangling side branches (which play the role of resonators) are grafted at $N$ equidistant sites, $N$ and $N^{\prime}$ being integers. The one-dimensional nature of the model requires that the cross sections of the two characteristic lengths be much less than the de Broglie wavelength, so as to neglect the quantum-size effects (or the subband structure). The stop bands originate both from the periodicity of the system and the states of the grafted branches. The width of the band gaps strongly depends on the contrast between the two characteristic lengths as well as on the numbers $N$ and $N^{\prime}$. On the other hand, the structure presented in Ref. 23 [called asymmetric serial loop structure (ASLS)] was made of asymmetric loops pasted together with segments of finite length, the loops playing the role of resonators. Such structure exhibits new features, in comparison with the CLS (Ref. 21) waveguide, for example, the existence of larger gaps, the avoidance of the constraint on the boundary condition at the end of the side branches, and the appearance of quasiquantized bands without inserting a defect.

The quasi-one-dimensional geometry presented in this paper is composed of a loop with dangling resonators; the whole structure is inserted between two semi-infinite leads (see Fig. 1). The two arms of the loop have different lengths $d_{2}$ and $d_{3}$. This results in symmetric or asymmetric loop depending on whether $d_{2}=d_{3}$ or $d_{2} \neq d_{3}$, respectively. The dangling resonators of lengths $d_{1}$ and $d_{4}$ are connected on both sides of the loop at the connection points of the two arms of the loop with the leads (see Fig. 1). Such a geometry combines the effects of the loop and of the dangling resonators and, consequently, may exhibit interesting features, in comparison with the CLS and ASLS waveguides, for example, the achievement of a large transmission stop band for a single loop and the appearance of sharp resonant electronic states inside the transmission gaps without introducing any defects in the structure. These features (which could be of potential interest in waveguide structures) are essentially due to the existence of the resonators connected with the loop (which is quite different from the case of CLS or ASLS). Indeed, in the case of an asymmetric loop, the occurrence of large stop bands in the transmission is related to the combinations of the zeros of transmission originating from the loop and from the dangling resonators. Also, the possibility of sharp resonant electronic states results from the internal resonances of the loop when such a resonance is located in the vicinity of a zero of transmission or squeezed between two zeros. On the other hand, in the case of a symmetric loop which does not display any zero in the transmission spectrum, the rate of transmission can only be depressed around the zeros associated with the dangling resonators by increasing their number. Also, a sharp resonant peak can be introduced in the transmission dips by appropriately tailoring the lengths of the dangling resonators.

This paper is organized as follows. In Sec. II, we give a brief review of the theoretical model used in this work as well as the elementary results of a single resonator and a single loop. These results are necessary for the understanding of the different structure proposed here, namely, a loop coupled to dangling resonators. Sections III and IV are devoted to numerical results of symmetric and asymmetric loops with dangling resonators, respectively. The conclusions are summarized in Sec. V.

\section{THEORETICAL MODEL}

To illustrate these original effects in a simple way, we describe the electronic propagation in the frame of freeparticle model in which $E=\left(\hbar^{2} k_{i}^{2} / 2 m_{i}\right)+V_{i}$, where $m_{i}, V_{i}$, and $k_{i}$ refer respectively to the effective mass, a constant potential, and a wave vector for medium with index $i$. We report on the results of the calculated transmission coefficients and phase or phase time as a function of the wave vector or energy. In this paper, we focus on homogeneous structures where media $1,2,3$, and 4 and the medium of the semiinfinite leads (see Fig. 1) are made of the same material, namely, GaAs. The material parameters are then $V_{i}=V$ $=0.0 \mathrm{meV}$ and $m_{i}=m=0.067 m_{0}$, where $m_{0}$ is the free electron mass.

Using the Green's function method, ${ }^{20}$ the expression giving the transmission function through the structure given in Fig. 1 can be obtained in the same way as in our previous works, ${ }^{21}$ namely,

$$
t=\frac{2 S_{1} S_{4}\left(S_{2}+S_{3}\right)}{\chi_{1}-j \chi_{2}},
$$

where

$$
\chi_{1}=2 S_{1} S_{4}\left(S_{2} C_{3}+S_{3} C_{2}\right)+S_{2} S_{3}\left(N C_{1} S_{4}+N^{\prime} C_{4} S_{1}\right)
$$

and

$$
\begin{aligned}
\chi_{2}= & S_{1} S_{4}\left(3 S_{2} S_{3}-2 C_{2} C_{3}+2\right)-\left(C_{3} S_{2}+C_{2} S_{3}\right) \\
& \times\left(N C_{1} S_{4}+N^{\prime} C_{4} S_{1}\right)-N N^{\prime} C_{1} C_{4} S_{2} S_{3} .
\end{aligned}
$$

$\mathrm{N}$ and $N^{\prime}$ are the numbers of dangling resonators on both sides of the loop, $C_{i}=\cos \left(k d_{i}\right), S_{i}=\sin \left(k d_{i}\right)(i=1,2,3,4)$, and $k=\frac{1}{\hbar} \sqrt{2 m E}$.

From the expression of $t$ [Eq. (1)], one can deduce the transmission coefficient

$$
T=\frac{4 S_{1}^{2} S_{4}^{2}\left(S_{2}+S_{3}\right)^{2}}{\chi_{1}^{2}+\chi_{2}^{2}}
$$

as well as the phase

$$
\varphi=\arctan \left(\chi_{2} / \chi_{1}\right)+\pi \Theta\left[S_{1} S_{4}\left(S_{2}+S_{3}\right)\right],
$$

where $\Theta$ means the Heaviside function. From Eqs. (4) and (5), one can notice that the transmission zeros are induced by the dangling resonators (i.e., $S_{1}=0$ or $S_{4}=0$ ) as well as the loop structure (i.e., $S_{2}+S_{3}=0$ ). When the expression $S_{1} S_{4}\left(S_{2}+S_{3}\right)$ changes sign at some energies denoted by $E_{n}$, then the phase [Eq. (5)] exhibits a jump of $\pi$.

Another interesting quantity is the first derivative of $\varphi$ with respect to the energy, which is related to the delay time taken by the electrons to traverse the structure. This quantity, called phase time, is defined by ${ }^{24,25}$ 

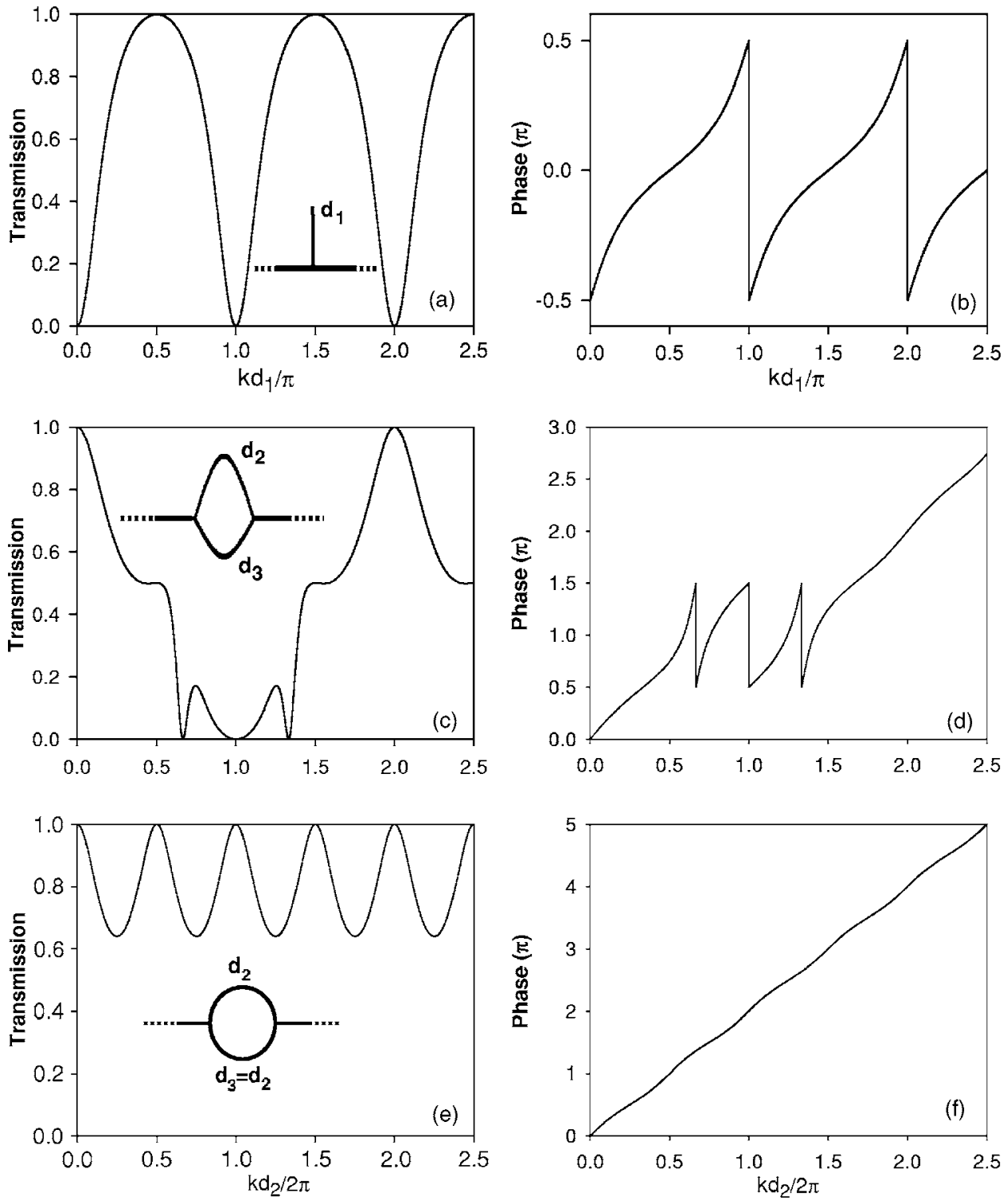

Because of the second term in the right-hand side of Eq. (7), one can deduce that $\tau_{\varphi} \neq \pi \Delta n(E)$ as $\tau_{\varphi}$ [Eq. (7)] may exhibit $\delta$ functions at the transmission zeros that do not exist in the variation of the DOS [Eq. (8)]. However, if the system does not exhibit transmission zeros or the expression $S_{1} S_{4}\left(S_{2}+S_{3}\right)$ does not change sign at some energies, then $\Theta\left[S_{1} S_{4}\left(S_{2}+S_{3}\right)\right]=0$ and $\tau_{\varphi}=\pi \Delta n(E)$. All these cases will be illustrated below in symmetric and asymmetric structures.

\section{CASE OF A SINGLE STUB AND A SINGLE LOOP: AN OVERVIEW}

Before addressing the problem of the whole structure cited above, let us first briefly recall the results of two particular cases necessary for the understanding of wave propagation in the structure shown in Fig. 1:

(i) If $d_{2}=d_{3}=0, N=1$, and $N^{\prime}=0$, we obtain the transmission function of a simple structure consisting of one resonator grafted on an infinite guide [see the inset of Fig. 2(a)]: 
$t=S_{1} /\left(S_{1}+j C_{1} / 2\right)$. This expression enables us to deduce the transmission coefficient $T=4 S_{1}^{2} /\left(4 S_{1}^{2}+C_{1}^{2}\right)$ and the phase $\varphi$ $=\pi \Theta\left(S_{1}\right)-\arctan \left(C_{1} / 2 S_{1}\right)$.

We can see that the transmission coefficient equals zero when $k d_{1}=l^{\prime} \pi$, where $l^{\prime}$ is a positive integer. The variation of $T$ versus the reduced wave vector $k d_{1} / \pi$ is reported in Fig. 2(a). $T$ is equal to zero for $k d_{1}$ being a multiple of $\pi$ and reaches its maximum value of 1 for $k d_{1}$ being an odd multiple of $\pi / 2$. The energies of the transmission zeros given by $E_{g}$ such as $E_{g}=\left(\hbar^{2} / 2 m\right)\left[l^{\prime} \pi / d_{1}\right]^{2}$ correspond to the eigenmodes of the grafted finite segment. This grafted segment behaves as a resonator and this simple composite system filters out the energies $E_{g}$. This phenomenon is related to the resonances associated with the finite additional path offered to the electronic wave propagation. The variation of the phase versus $k d_{1} / \pi$ [Fig. 2(b)] shows an abrupt change of $\pi$ at the transmission zeros, and therefore the corresponding phase time is different from the DOS as mentioned above.

(ii) If $N=N^{\prime}=0$, we obtain the transmission function of a single loop inserted between two leads [see the inset of Fig. 2(c)],

$$
t=\frac{2\left(S_{2}+S_{3}\right)}{2\left(C_{2} S_{3}+C_{3} S_{2}\right)-j\left(3 S_{2} S_{3}-2 C_{2} C_{3}+2\right)},
$$

which gives the transmission coefficient

$$
T=\frac{4\left(S_{2}+S_{3}\right)^{2}}{8+5 S_{2}^{2} S_{3}^{2}-4 S_{2} S_{3} C_{2} C_{3}-8 C_{2} C_{3}+12 S_{2} S_{3}}
$$

and the phase

$$
\begin{aligned}
\varphi= & \pi \Theta\left(S_{2}+S_{3}\right)+\arctan \left[\left(3 S_{2} S_{3}-2 C_{2} C_{3}+2\right) / 2\left(S_{2} C_{3}\right.\right. \\
& \left.\left.+S_{3} C_{2}\right)\right] .
\end{aligned}
$$

In this case, the transmission zeros are given by $S_{2}+S_{3}$ $=0$ or, equivalently,

$$
k\left(d_{2}+d_{3}\right)=2 n \pi
$$

and

$$
k\left(d_{2}-d_{3}\right)=\left(2 n^{\prime}+1\right) \pi,
$$

where $n$ and $n^{\prime}$ are integers. One can notice that as far as $d_{2} \neq d_{3}$, the transmission vanishes and changes sign at different values of $k d_{2} / 2 \pi$, as illustrated in Fig. 2(c) for $d_{2}=2 d_{3}$. The two transmission zeros at $k d_{2} / 2 \pi=2 / 3$ and $4 / 3$ are obtained from Eq. (12) for $n=1$ and $n=2$, whereas the transmission zero at $k d_{2} / 2 \pi=1$ is obtained from Eq. (13) for $n^{\prime}$ $=0$. The associated phase curve [Fig. 2(d)] exhibits, as predicted, a phase jump at the transmission zeros, and therefore the phase time is different from the DOS as explained before.

In the particular case of a symmetric loop [i.e., $d_{2}=d_{3}$, see the inset of Fig. 2(e)], the transmission coefficient and the phase become respectively $T=16 /\left(25-9 C_{2}^{2}\right)$ and $\varphi$ $=\arctan \left(3 S_{2} / 4 C_{2}\right)$. One can notice that in this case, the transmission [Fig. 2(e)] does not present any zeros, and therefore the phase increases monotonically, as illustrated in Fig. 2(f). In this case, the phase time is equivalent to the DOS as explained above.

Let us now discuss the geometry of a loop with dangling resonators. We shall address the problem of the effect of dangling resonators respectively on a symmetric loop and on an asymmetric loop.

\section{EFFECT OF DANGLING RESONATORS ON SYMMETRIC LOOP}

In the case of a symmetric loop (i.e., $d_{2}=d_{3}$ ) with dangling resonators [see the inset of Fig. 3(a)], the expression of the transmission function [Eq. (1)] becomes

$$
t=\frac{4 S_{1} S_{4}}{4 S_{1} S_{4} C_{2}+S_{2}\left(N C_{1} S_{4}+N^{\prime} C_{4} S_{1}\right)+j\left[-5 S_{2} S_{1} S_{4}+2 C_{2}\left(N C_{1} S_{4}+N^{\prime} C_{4} S_{1}\right)+N N^{\prime} C_{1} C_{4} S_{2}\right]} .
$$

Equation (14) clearly shows that the transmission zeros are due only to the dangling resonators (i.e., when $S_{1}=0$ or $S_{4}=0$ ). Figure 3(a) gives the transmission coefficient for a symmetric loop in the presence of two identical dangling resonators (i.e., $N=N^{\prime}=1$ and $d_{1}=d_{4}=0.5 d_{2}$ ). One can notice that the transmission coefficient presents well defined dips induced by the grafted resonators. These dips transform into large transmission gaps when the number of resonators increases as illustrated in Figs. 3(c) and 3(e) for $N=N^{\prime}=2$ and $N=N^{\prime}=3$, respectively. It is worth mentioning that because of the existence of two resonators, one can expect two phase drops of $\pi$ (i.e., $2 \pi$ ) at the transmission zeros given by $S_{1}$ $=S_{4}=0$ (i.e., $k d_{2} / 2 \pi=1$ ). However, one can see in Figs. 3(b), $3(\mathrm{~d})$, and 3(f) that the phase presents only a phase drop of $\pi$. This is due to the existence of a resonant state with zero width at this value of $k d_{2} / 2 \pi$, which induces a phase jump of $+\pi$; this resonance collapses when $d_{1}=d_{4}$ is taken exactly equal to $0.5 d_{2}$. To enlarge this resonance, we have to take $d_{1}$ and $d_{4}$ slightly different from $0.5 d_{2}$. Indeed, at $k d_{2} / 2 \pi=1$, the expression of the transmission function [Eq. (14)] becomes

$$
t=\frac{S_{1} S_{4}}{S_{1} S_{4}+j \sin \left[k\left(d_{1}+d_{4}\right)\right]} .
$$

So, if $k\left(d_{1}+d_{4}\right)=m \pi$ but $k d_{1} \neq m_{1} \pi$ and $k d_{2} \neq m_{2} \pi$ ( $m$, $m_{1}$, and $m_{2}$ are integers), one obtains a resonance that reaches unity (i.e., $t=1$ ). An example corresponding to this situation is given in Fig. 4(a), where $d_{1}=0.46 d_{2}$ and $d_{4}$ $=0.54 d_{2}\left(\right.$ with $\left.d_{1}+d_{4}=d_{2}\right)$. One can notice that the resonance 

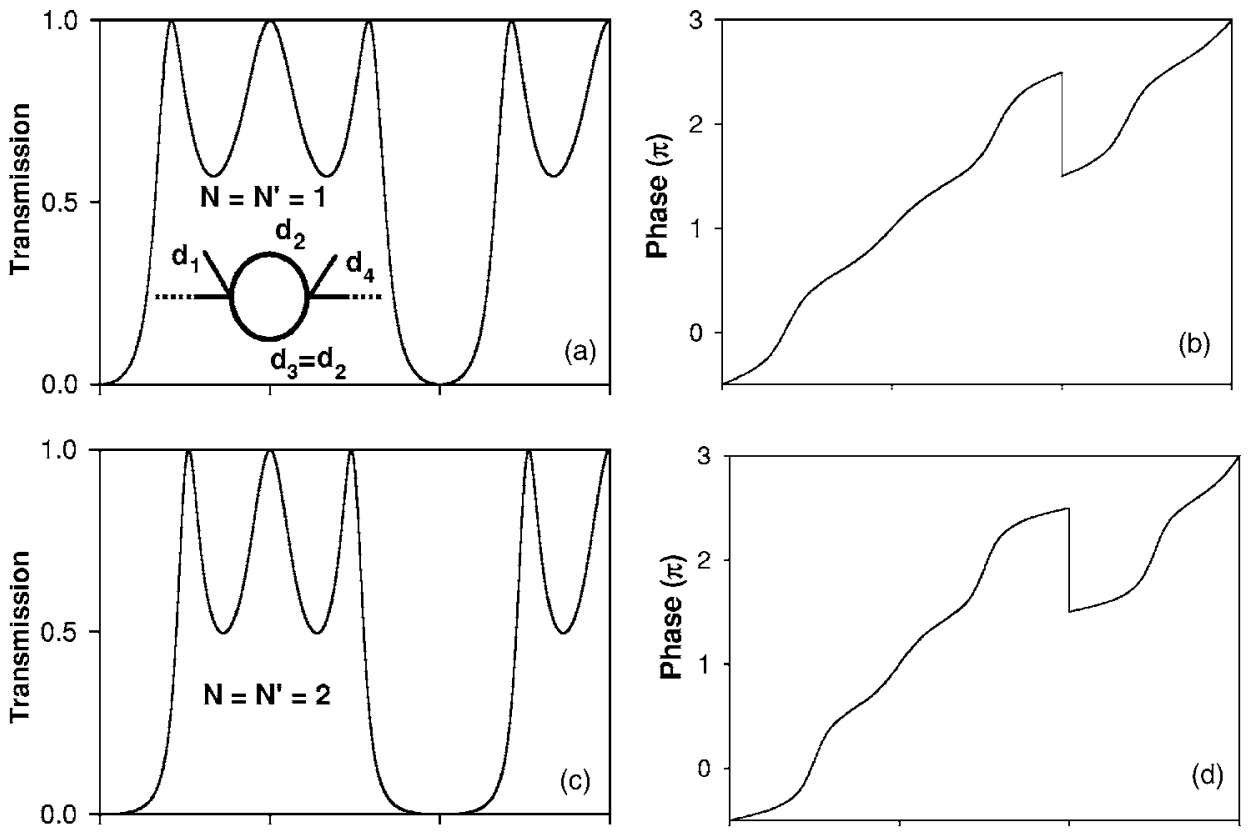

FIG. 3. Left panel: [(a), (c), and (e)] The same as in Fig. 2(e) but for a single symmetric loop in the presence of resonators: (a) $N$ $=N^{\prime}=1$, (b) $N=N^{\prime}=2$, and (c) $N$ $=N^{\prime}=3$. Right panel: The same as in the left panel but for the variation of the phase.
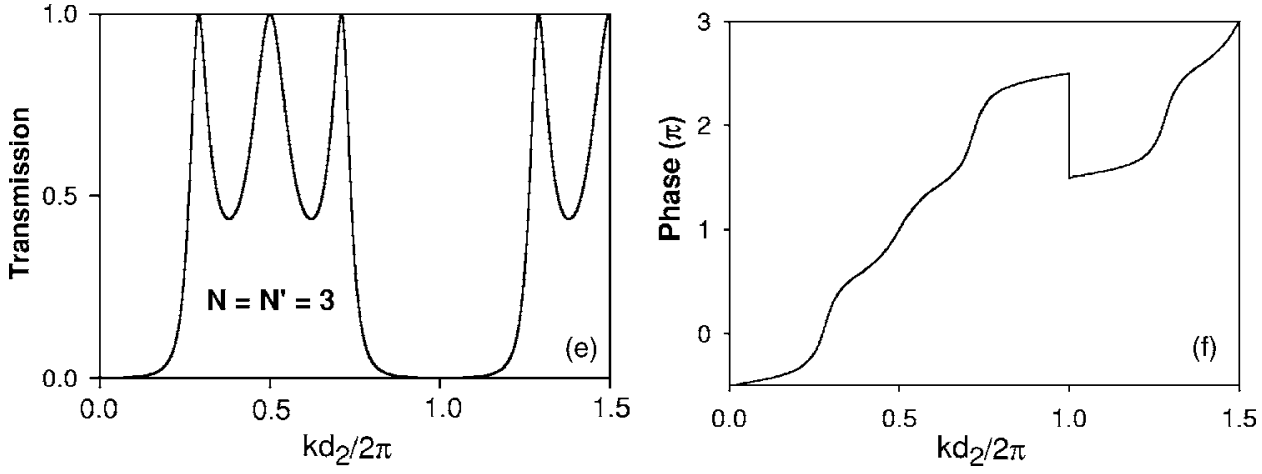

at $k d_{2} / 2 \pi=1$ is squeezed between two zeros [indicated by solid circles on the abscissa of Fig. 4(a)] induced by the surrounding resonators, as also illustrated in the curve of the variation of the phase [Fig. 4(b)]. The width of this resonance increases as far as $d_{1}$ and $d_{4}$ deviate from $0.5 d_{2}$ (see below). In the particular case where $k d_{1}=m_{1} \pi$ and $k d_{2}$ $=m_{2} \pi$, the numerator and denominator of $t$ [Eq. (15)] vanish altogether. In this case, the resonance as well as the two zeros induced by the resonators fall at the same position, then the resonance collapses, the transmission coefficient vanishes, and the phase drops by $\pi$, as shown in Figs. 3(b), 3(d), and 3(f).

The resonance in Fig. 4(a) shows the same characteristics as a Fano resonance but with two zeros of transmission around the resonance instead of one, as is usually the case. ${ }^{9}$ Indeed, one can obtain an approximate analytical expression for the transmission function [Eq. (14)] in the vicinity of the resonance. A Taylor expansion around $k d_{2}=2 \pi$ (i.e., $k d_{2}$ $=2 \pi+\varepsilon$ with $\varepsilon / 2 \pi \ll 1$ ) enables us to obtain

$$
t=\frac{\zeta \zeta^{\prime}}{\varepsilon^{2}+\zeta \zeta^{\prime}+j \varepsilon\left(3-\zeta \zeta^{\prime}\right)},
$$

where $\zeta=2 \Delta+\varepsilon(1+\Delta / \pi), \zeta^{\prime}=-2 \Delta+\varepsilon(1-\Delta / \pi)$, and $\Delta$ is the detuning of $d_{1}$ and $d_{4}$ from $0.5 d_{2}$ [i.e., $\Delta=2 \pi\left(0.5-d_{1} / d_{2}\right)$ $\left.=2 \pi\left(-0.5+d_{4} / d_{2}\right)\right]$.
From Eq. (16), one can show that the transmission coefficient $T$ can be written (following the Fano line shape ${ }^{9}$ ) in the form

$$
T=A \frac{(\varepsilon+q \Gamma)^{2}(\varepsilon-q \Gamma)^{2}}{\varepsilon^{2}+\Gamma^{2}},
$$

where $A=\left(1-\Delta^{2} / \pi^{2}\right)^{2} /(3+4 \Delta)^{2} . \Gamma=4 \Delta^{2} /\left(3+4 \Delta^{2}\right)$ characterizes the width of the resonance falling at $\varepsilon=0 . q=(3$ $\left.+4 \Delta^{2}\right) / 2 \Delta(1+\Delta / \pi)$ is the coupling parameter, which gives qualitatively the interference between the bound states and the propagating continuum states. ${ }^{9,10}$ One can notice that when increasing $\Delta, \Gamma$ increases and $q$ decreases. The results of the approximate expression [Eq. (17)] are given in the inset of Fig. 4(a) by the open circles. These results are in accordance with the exact ones (solid lines) and clearly show that the resonance is of Fano type with $q \simeq 7$ and width $2 \Gamma$ $\simeq 0.15$. The commonly studied Fano resonances are asymmetric because of the presence of only one transmission zero near the resonance; in addition, an impurity is often introduced in one of the two arms of the loop in order to create the resonance state. The above calculation shows that without introducing any perturbation in the arms of the loop, one can find a well defined symmetric Fano resonance with a width $2 \Gamma$ and a coupling parameter $q$ that can be adjusted by 

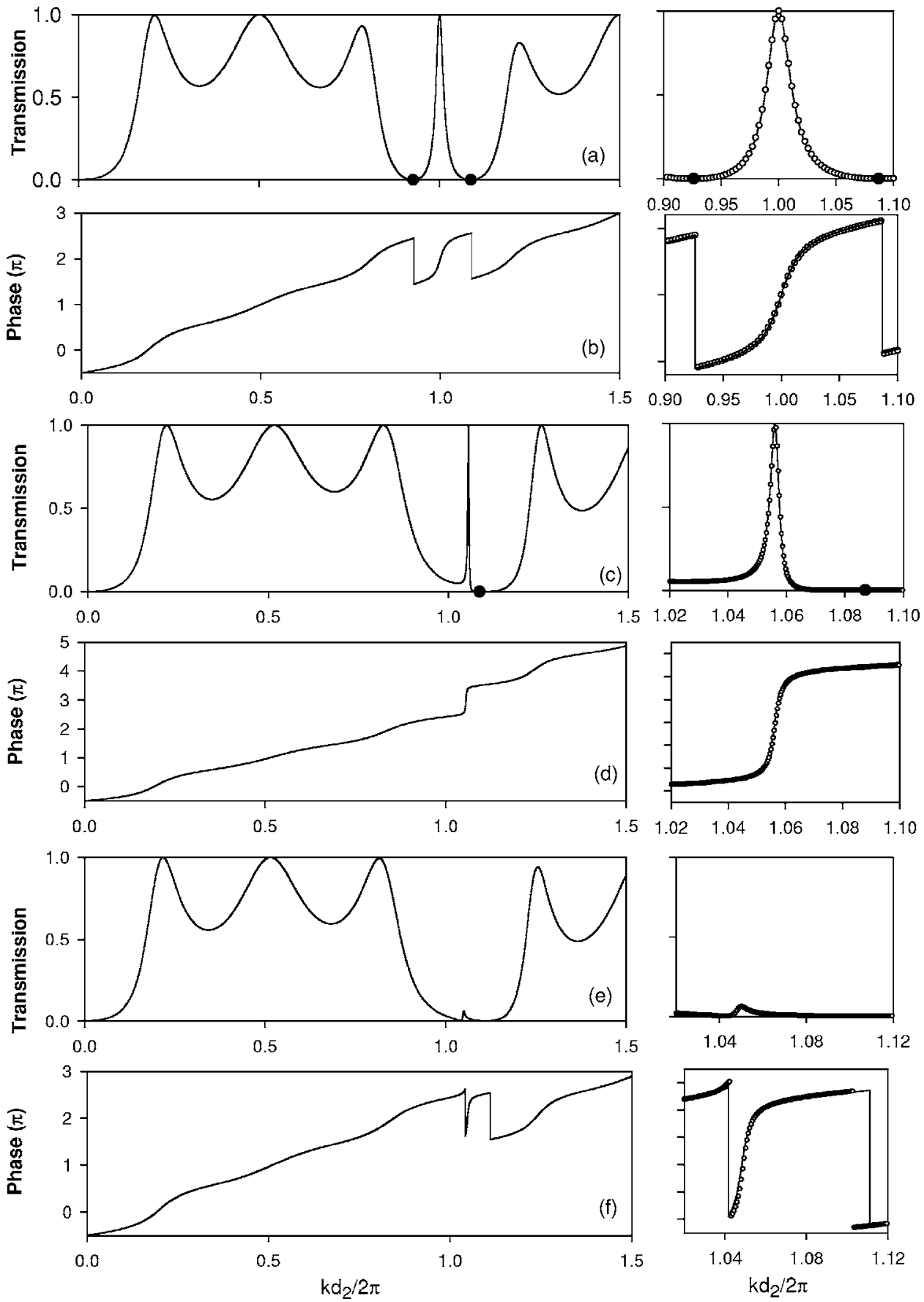

FIG. 4. (a) The same as in Fig. 3(a) but the lengths of the resonators are taken such that $d_{1}=0.46 d_{2}$ and $d_{4}=0.54 d_{2}$ and $N=N^{\prime}=1$. The solid circles on the abscissa axis indicate the positions of the transmission zeros induced by the dangling resonators on both sides of the resonance. The inset gives the approximate results (open circles) around the resonance. (b) The same as in (a) but for the variation of the phase. (c) The same as in (a) but the resonators are taken to be of identical lengths $d_{1}=d_{4}=0.46 d_{2}$. (d) The same as in (c) but for the variation of the phase. [(e) and (f)] The same as in (a) and (b) but the resonators are taken such that $d_{1}=0.48 d_{2}$ and $d_{4}=0.45 d_{2}$.

tailoring the lengths of the resonators (i.e., $\Delta$ ) surrounding the loop. Equation (16) also enables us to deduce an approximate expression for the phase as

$$
\varphi=\pi \Theta(\zeta)+\pi \Theta\left(\zeta^{\prime}\right)-\arctan \left[\varepsilon\left(3-\zeta \zeta^{\prime}\right) /\left(\varepsilon^{2}+\zeta \zeta^{\prime}\right)\right] .
$$

This function is plotted by the open circles in the inset of Fig. 4(b) and clearly show two abrupt phase changes of $\pi$ at $\zeta=0$ and $\zeta^{\prime}=0$ (i.e., $\varepsilon= \pm q \Gamma$ ), in accordance with the exact results (solid line).

One can also create an asymmetric Fano resonance by adjusting the transmission zeros on only one side of the resonance; this can be obtained by considering a symmetric structure where the resonators are supposed to be identical with lengths slightly different from $0.5 d_{2}$. This is shown in Fig. 4(c) for $d_{1}=d_{4}=0.46 d_{2}$ and $N=N^{\prime}=1$. Indeed, an ana- 
lytical Taylor expansion around $k d_{2}=2 \pi$ enables us to write the transmission function [Eq. (14)] as

$$
t=\frac{4 \xi^{2}}{(\xi+j)(\varepsilon+4 \xi-j \varepsilon \xi)},
$$

where $\xi=\Delta+\varepsilon(1+\Delta / \pi) / 2$ and $\Delta$ is the detuning of the lengths of the two resonators from $0.5 d_{2}$ [i.e., $\Delta=2 \pi\left(d_{1} / d_{2}\right.$ $-0.5)]$.

From the expression of $t$ [Eq. (19)], one can deduce the following Fano line-shape transmission coefficient:

$$
T=B \frac{1}{1+\xi^{2}} \frac{\left(\varepsilon-\varepsilon_{R}+q \Gamma\right)^{4}}{\left(\varepsilon-\varepsilon_{R}\right)^{2}+\Gamma^{2}} \simeq B \frac{\left(\varepsilon-\varepsilon_{R}+q \Gamma\right)^{4}}{\left(\varepsilon-\varepsilon_{R}\right)^{2}+\Gamma^{2}},
$$

where $B=(1+\Delta / \pi)^{4} / 9(1+2 \Delta / 3 \pi)^{2}$.

$\Gamma=4 \Delta^{2}(1-2 \Delta / 3 \pi) / 27(1+2 \Delta / 3 \pi)^{2}$ and $\varepsilon_{R}=-4 \Delta / 3(1$ $+2 \Delta / 3 \pi)$ characterize the width and the position of the resonance, respectively, whereas $q=9(1+2 \Delta / 3 \pi) / 2 \Delta(1$ $-2 \Delta / 3 \pi)$ is the Fano parameter. One can notice that the resonance shifts slightly from $k d_{2}=2 \pi$ and its width is small as compared to the preceding case; this is in accordance with the numerical results of Figs. 4(a) and 4(c). Also, $q$ increases when $\Delta$ decreases and tends to infinity when $\Delta$ vanishes. In this case, the resonance falls at $\varepsilon_{R}=0$ and its width $2 \Gamma$ reduces to zero [Fig. 3(a)], as expected. The results of the approximate expression [Eq. (19)] are sketched (open circles) in the inset of Fig. 4(c) for $\Delta=2 \pi\left(d_{1} / d_{2}-0.5\right)$ $=-0.08 \pi$ (i.e., $d_{1} / d_{2}=0.46$ ). These results are in accordance with the exact ones (solid lines) and clearly show that the resonance is of Fano type with $q \simeq 16$ and width $2 \Gamma$ $\simeq 0.022$. Concerning the evolution of the phase of the electrons in this structure, one can notice from Eq. (14) that the numerator of the transmission function $t$ vanishes when $S_{1}$ $=S_{4}=0$ \{or equivalently $\xi=0$ in the approximate result [Eq. (19)]\} at $k d_{2} / 2 \pi=d_{2} / 2 d_{1}=1.086$, indicated by a filled circle on the abscissa axis of Fig. 4(c). However, it does not change sign, as it is composed of a square value of $S_{1}\{\xi$ in the approximate result [Eq. (19)]\}. This is clearly shown in Fig. 4(d), where the phase does not display any abrupt change of $\pi$ even though the transmission coefficient vanishes [see also the inset of Fig. 4(d), where no phase drops appear in the exact and approximate results]. This result has been pointed out recently ${ }^{26}$ in two-dimensional quantum dots by different theoretical models. It is worth mentioning that in this case the derivative of the phase with respect to the energy (i.e., the phase time) is identical to the DOS, as explained in Sec. II. To end this section, let us note that a small deviation of $d_{1}$ and $d_{4}$ from the values chosen above may considerably reduce the intensity of the resonance, as shown in Fig. 4(e) for $d_{1}=0.48 d_{2}$ and $d_{4}=0.45 d_{2}$. This result shows the sensitivity of the resonances to the lengths of the surrounding resonators. The corresponding phase variation [Fig. 4(f)] increases smoothly between two abrupt phase drops of $\pi$ induced by the two dangling resonators, showing a signature of the existence of a resonant state. The Taylor expansion of the transmission coefficient giving the approximate results in the inset of Figs. 4(e) and 4(f) is more complicated and we avoid presenting it here.
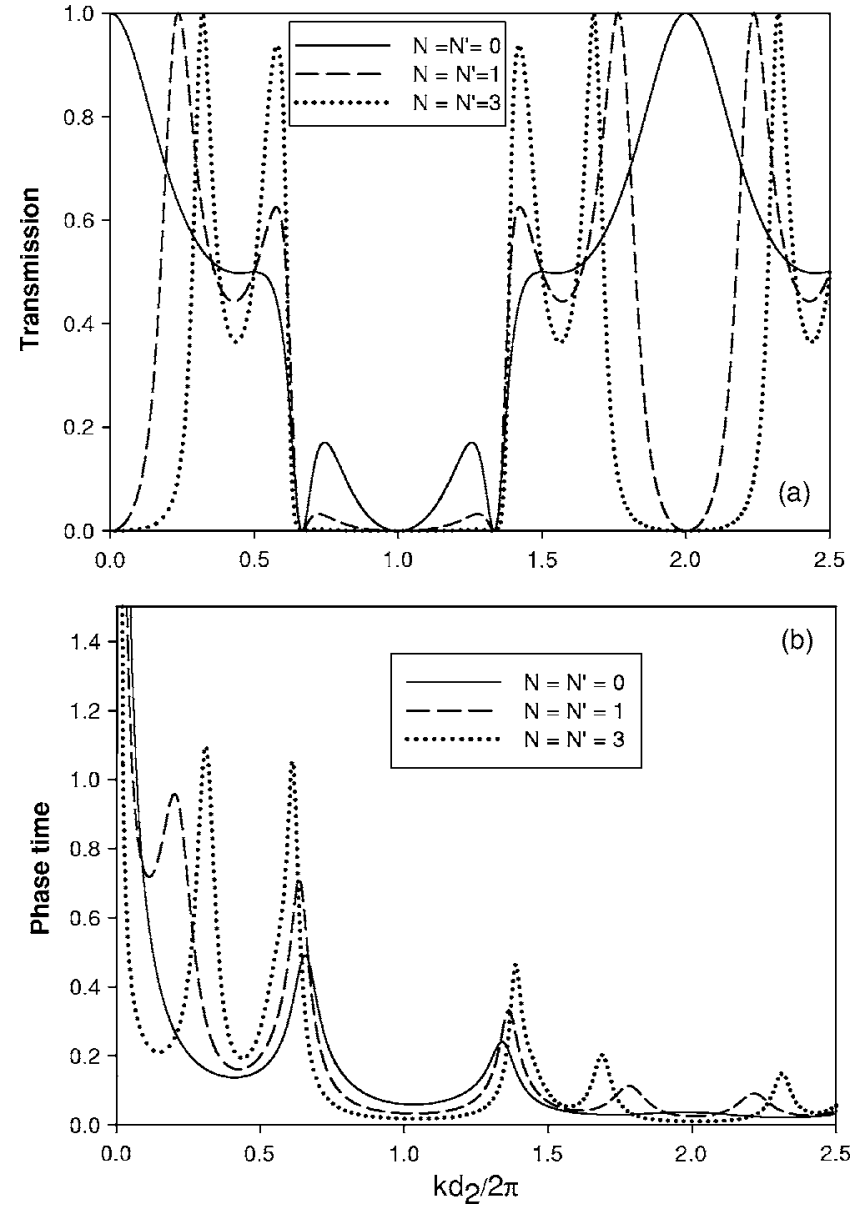

FIG. 5. (a) Variations of the transmission coefficient vs reduced wave vector $k d_{2} / 2 \pi$ through a single asymmetric loop with dangling resonators for $d_{2}=2 d_{3}$. The calculation is performed for $N=N^{\prime}=0$ (solid curve), $N=N^{\prime}=1$ (dashed curve), and $N=N^{\prime}=3$ (dotted curve). (b) Variations of the phase time (in units of $2 m d_{2}^{2} / \hbar$ ) vs the reduced wave vector for the structure described in (a).

\section{EFFECT OF DANGLING RESONATORS ON ASYMMETRIC LOOP}

We have chosen the same asymmetric loop as in Fig. 2(c) (i.e., $d_{3}=0.5 d_{2}$ ) in the presence of two identical dangling resonators of lengths $d_{1}=d_{4}=0.5 d_{2}$. These results are plotted in Fig. 5(a) by the dashed and dotted lines for $N=N^{\prime}=1$ and $N=N^{\prime}=3$, respectively. The curve associated with the loop without resonators $\left(N=N^{\prime}=0\right)$ is redrawn for the sake of comparison (solid line). One can notice that one obtains a well defined gap around $k d_{2} / 2 \pi=1$; its width remains almost constant but becomes deeper when increasing the number of resonators. This transmission gap has no analog for a symmetric loop. This system could be useful in constructing a reflector for electrons, in analogy to photons in photonic band-gap crystals. The presence of resonators transforms the resonance at $k d_{2} / 2 \pi=2$ for $N=N^{\prime}=0$ into a dip, which can be enlarged when the number of resonators increases in the same way as for a symmetric loop (Fig. 3). In Fig. 5(b), we have given the variation of the phase (or delay) time [Eq. (6)] for the same parameters as in Fig. 5(a). This quantity gives information on the time spent by the electron inside the 

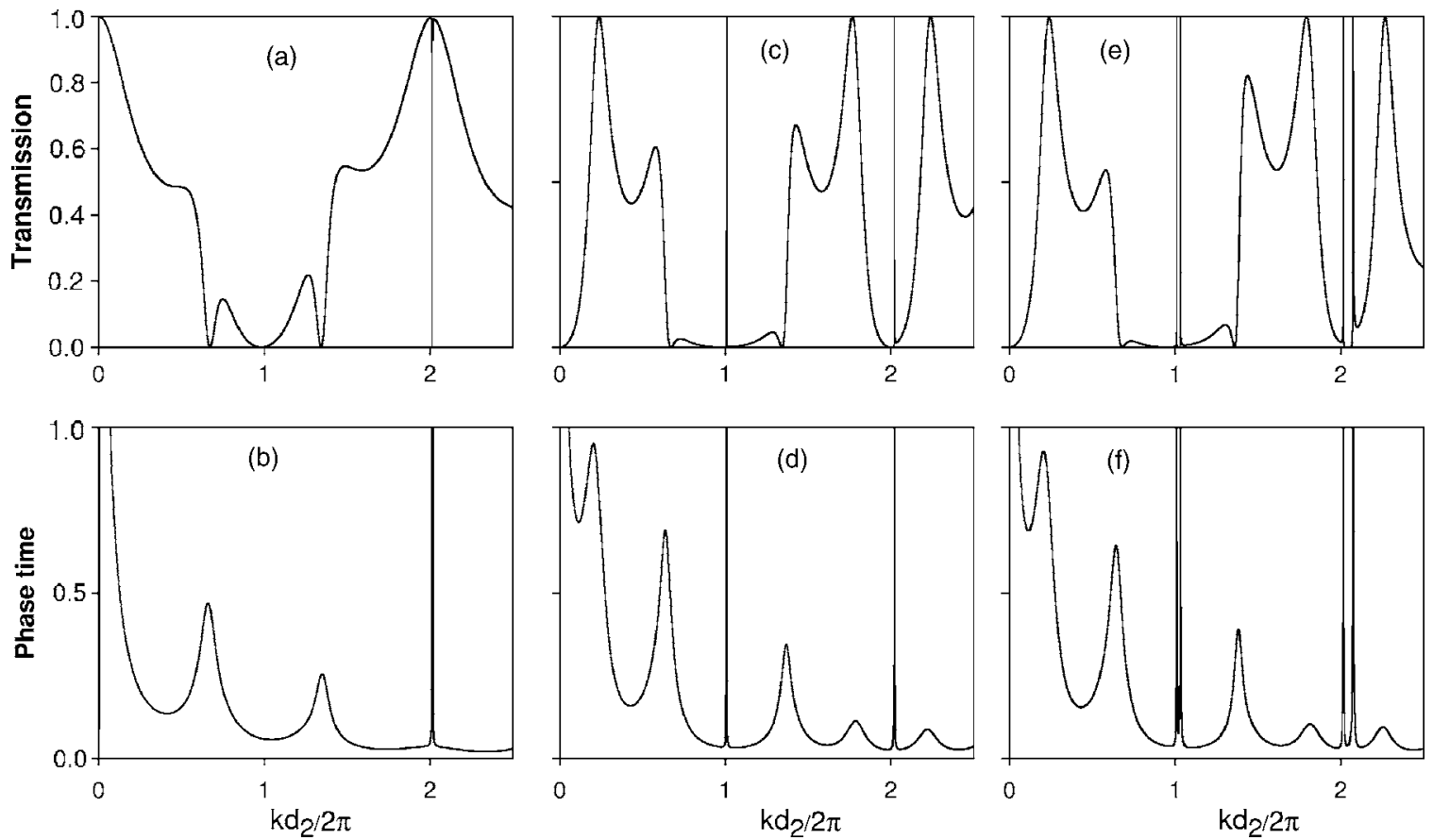

FIG. 6. Top panel (a) Transmission spectrum vs reduced wave vector for a single asymmetric loop without dangling resonators $\left(N=N^{\prime}=0\right)$. The geometrical parameter is considered to be $d_{3}=0.49 d_{2}$. (c) The same as in (a) but in the presence of two dangling resonators on both sides of the loop $\left(N=N^{\prime}=2\right)$ with lengths $d_{1}=d_{4}=0.5 d_{2}$. (e) The same as in (c) but with $d_{3}=0.49 d_{2}$ and $d_{1}=d_{4}=0.47 d_{2}$. Bottom panel: [(b), (d), and (f)] The same as in the top panel but for the phase time.

structure (cavity) before its transmission. One can notice that the phase time is strongly reduced in the transmission gap regions, in particular, when the number of dangling resonators increases. It is worth noting that the transmission zeros give rise to delta functions in the phase time [Eq. (7)]. To observe these delta peaks, the phase time should be enlarged around the transmission zeros by taking into account the absorption in the system ${ }^{27}$ (see Fig. 7 discussed below).

In order to introduce sharp resonances in the forbidden bands, we have kept (Fig. 6) the same structure as in Fig. 5 but slightly changed the lengths of the arms constituting the loop and the dangling resonators. In Fig. 6(a), we have considered the asymmetric loop without resonators (i.e., $N=N^{\prime}$ $=0)$ and $d_{3} / d_{2}=0.49$. In this case, a sharp zero of transmission (dip) appears at $k d_{2} / 2 \pi \simeq 2$ according to Eq. (12) for $n=3$. Such a very narrow stop band could be useful in constructing a rejecting signal device. This dip disappears when $d_{3} / d_{2}$ is exactly equal to 0.5 , as shown in Fig. 5(a) (solid line). Figure 6(b) shows the corresponding phase time as a function of $k d_{2} / 2 \pi$. A strong peak occurs in association with the sharp zero of transmission at $k d_{2} / 2 \pi \simeq 2$. These results clearly show that the absence of dangling resonators on both sides of the loop does not make it possible to create sharp resonances inside the transmission gaps. This is also the case when only one resonator is grafted on one side of the loop. However, when one connects two identical resonators $(N$ $=N^{\prime}=2$ ) (of lengths $d_{1}=d_{4}=0.5 d_{2}$ ) on both sides of the loop at the points of connection of the two arms of the asymmetric loop, leaving all the other parameters as in Fig. 6(a), we obtain Fig. 6(c), which presents now an interesting sharp mode inside each transmission gap. The creation of these modes is related to the resonances associated with the finite additional path offered to the electronic wave propagation. This feature could be used as a transmission filter for an electronic signal. In Fig. 6(d), there are two distinct peaks in the phase time calculation, one associated with the mode created at $k d_{2} / 2 \pi \simeq 1$ and the other one with the mode that occurs at $k d_{2} / 2 \pi \simeq 2$ [see Fig. 6(c)]. These calculations show an enhancement in the electron capture time inside the structure for certain values of energy. It is worth noting that the sharp resonances in Fig. 6(c) are of Fano type as for symmetric loop. However, because of the complexity of the calculations, we did not give here the approximate expressions showing this phenomenon.

Now, if different characteristic lengths for $d_{2}, d_{3}$, and $d_{1}$ $=d_{4}$ are imposed on the structure, two very closed localized states can be observed in the transmission gaps (without introducing any defects in the structure). This is illustrated in Fig. 6(e), where we have reported the transmission coefficient versus $k d_{2} / 2 \pi$ for the same structure as in Fig. 6(c) but with $d_{3} / d_{2}=0.49$ and $d_{1} / d_{2}=0.47$. The corresponding phase time variation is reported in Fig. 6(f), showing a strong localization of the modes falling inside the gaps. These results show that by tailoring the lengths of the different wires constituting the structure, one can create large gap reflectors with or without defect modes that may play the role of filters.

An interesting quantity that may considerably affect the amplitude and the phase of the transmission through such structures is the absorption. This quantity often exists in experiments as an effect of temperature, scatterers, or electronic correlations, as well as magnetic fields applied on the quantum dot together with a plunger gate voltage. ${ }^{9}$ In this case, the system becomes an open interferometer with no conservation of current. Some theoretical models ${ }^{28}$ have used 

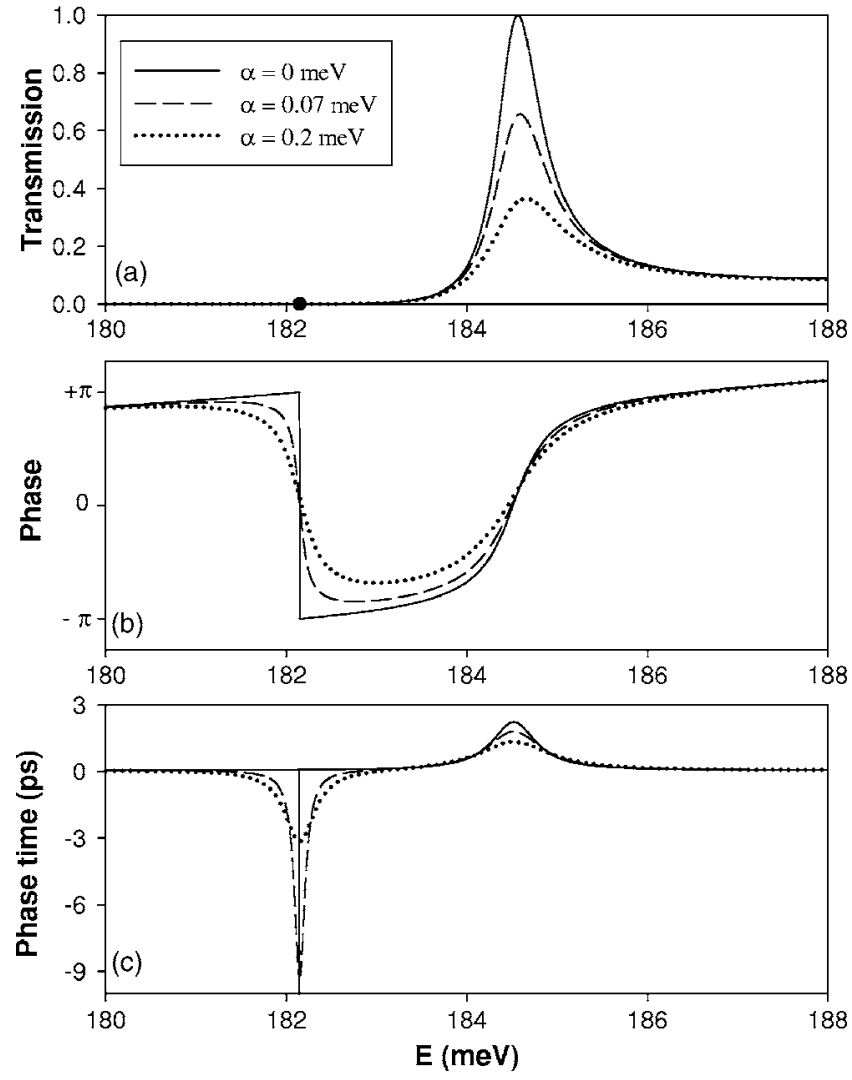

FIG. 7. (a) Transmission vs energy (meV) for a single asymmetric loop with dangling resonators $\left(N=N^{\prime}=1\right)$. The geometrical parameters are $d_{2}=100 \mathrm{~nm}, d_{3}=49 \mathrm{~nm}$, and $d_{1}=d_{4}=50 \mathrm{~nm}$. The absorption is introduced in the system by adding an imaginary part $\alpha$ to energy: $\alpha=0 \mathrm{meV}$ (solid curve), $\alpha=0.07 \mathrm{meV}$ (dashed curve)s, and $\alpha=0.2 \mathrm{meV}$ (dotted curve). The solid circle indicates the position of the transmission zero. (b) The same as in (a) but for the phase. (c) The same as in (a) but for the phase time.

additional leads grafted at different positions on the structure to model losses of electronic current, thus breaking unity. In this work, we have introduced the dissipation in the system by adding a small imaginary part $\alpha$ to the energy (i.e., $E$ $+j \alpha$ ). In Fig. 7, we give an illustrative example of a Fano resonance in an asymmetric loop composed of different wires such that $d_{1}=d_{4}=50 \mathrm{~nm}, d_{2}=100 \mathrm{~nm}$, and $d_{3}=49 \mathrm{~nm}$. For $\alpha=0$ (no dissipation), the transmission coefficient versus the energy exhibits a resonant peak around $E=184.56 \mathrm{meV}$ (solid line) and a transmission zero around $E$ $=182.145 \mathrm{meV}$ [indicated by a solid circle on the abscissa axis in Fig. 7(a)]. The corresponding phase [solid line in Fig. 7(b) ] increases smoothly by $\pi$ around the resonance and presents an abrupt phase drop of $\pi$ at the transmission zero. The variation of the phase time [solid line in Fig. 7(c)] shows a delay time of almost $2.24 \mathrm{ps}$ around the resonance and a delta peak at the transmission zero. In the presence of dissipation (dashed and dotted lines in Fig. 7), the intensity of the resonance decreases significantly even for a small absorption such as $\alpha=0.07 \mathrm{meV}$ (dashed curves). The corresponding phase and phase time are considerably affected around the energies of the resonance peak and the transmission zero. In particular, the absorption diminishes the phase time but en- ables the observation of negative delta phase times around the transmission zero by widening it [Fig. 7(c)]. Let us mention that negative phase time corresponds to negative group velocity $v_{g}$, since the latter quantity is related to the phase time $\tau$ by $v_{g}=L / \tau$, where $L$ is the length of the structure. ${ }^{27}$

Negative phase time or equivalently negative group velocity corresponds to the case in which the peak of tunneled pulse exists before the peak of the incident pulse has entered the sample. This occurs through the forbidden transmission region where the dispersion is anomalous. Indeed, in dielectric materials, it is well known that in the presence of absorption, the refraction index (or equivalently the phase of the transmission) may take a steep drop, ${ }^{29}$ resulting in an anomalous dispersion and, consequently, in a light pulse propagation at group velocity faster than $c$ or even negative. ${ }^{27,30-34}$ In all these works, it was clearly pointed out that such superluminal behavior is not at odds with either causality or Einstein theory of special relativity, but it exclusively results from interference between the different frequency components of the pulse in an anomalous dispersion region. ${ }^{35,36}$ However, the formal analogy between the Schrödinger equation and the Helmholtz equation for electromagnetic wave enables one to correlate the results for optical experiments to that for electrons. Therefore, negative electron travel time can be understood in the same manner as for electromagnetic waves.

\section{SUMMARY AND REMARKS}

In conclusion, we have presented a 1D monomode structure exhibiting large electronic stop bands. A theoretical investigation of the electronic transmission power through a 1D single symmetric (or asymmetric) loop with dangling resonators using a Green's function method is presented. Compared to other 1D networks such as CLS waveguides, the observed stop bands in the single symmetric (or asymmetric) loop with dangling resonators are significantly larger. The existence of the stop bands in the spectrum is attributed to the conjugation of the zero transmission associated with the dangling resonators and the asymmetry of the loop. In these systems, the stop band width is controlled by the geometrical parameters. Numerical results on localized modes in nonperturbed waveguide were also reported. These localized states appear as Fano resonances of strong amplitude in the transmission spectra. Asymmetric and symmetric Fano resonances that may lie near the vicinity of a transmission zero or be squeezed between two transmission zeros are obtained and analyzed. By tailoring the lengths of the different wires constituting the structure, one can introduce one or two resonances inside the transmission gaps. The phase time calculations are, in general, different from the density of states, except for particular structures where the numerator of the transmission function does not vanish or vanishes but without changing sign. In this case, the phase time and the DOS are equivalent. The localized states give rise to well defined peaks in the phase time. The effect of absorption on the amplitude and the phase time of the transmission has been reviewed.

Through our analysis, we use the one-channel transport model for the quantum-wire network. The single-channel 
model system is an idealization of the real quantum wires with finite width and provides a good approximation for the network of the wires with a narrow width at low temperature. In this case, only the lower subband is filled. If the cross sections of the wires are of nonzero width and at higher temperatures, the studies of the conductance of the quantum waveguides should employ the two-dimensional model. ${ }^{37-40}$ In such a study, the multimode effect and the matching of the transverse modes are necessarily considered. The lateral confinement in the wires gives rise to a quantization of the conductance $G$ in units of $2 e^{2} / \mathrm{h}$, which reflects the number of active channels in the transport measurement. ${ }^{37,38}$ In addition to the Fabry-Pérot-like conductance oscillations within the $2 e^{2} / \mathrm{h}$ quantized plateaus, it was shown that the existence of other geometries connected to the wires such as a single stub $^{40}$ may give rise to a complicated spectrum in the second subband with an irregular pattern of maxima and minima in comparison with the periodic feature in the first subband (see, for example, Figs. 8 and 11 in Ref. 40). The transmission zeros induced by the stub strongly depend on the width of the waveguide along which the stub is attached. ${ }^{41}$ Also, the existence of crosses $^{42}$ and bends ${ }^{43}$ in the structure may also result in bound states associated with the cross sections of these contact points.

Another important effect that may influence the transmission spectrum in quantum wires is the electron-electron interaction. ${ }^{44}$ Indeed, it was shown recently ${ }^{45}$ that a model incorporating Coulomb interaction gives quite good fits of the experimental conductance spectrum associated with Fano resonances in a one lead quantum dot. Also, it was argued theoretically, ${ }^{46}$ using a tight-binding model and a selfconsistent calculation, that Fano resonances shift as function of the applied gate voltage when electron-electron interaction is taken into account. These results are found in accordance with the experimental work of Buks et al. ${ }^{47}$ However, it was shown that electron interaction does not affect much persistent current measurements in a single semiconductor loop. ${ }^{13}$
In addition, the spin-orbit interaction, the so-called Rashba effect, in one-dimensional rings and stubs, has been considered recently ${ }^{48-50}$ by adding a spin-related term to the Hamiltonian. In particular, it was shown that the transmission spectrum splits into two spectra corresponding to outgoing electrons similar as or opposite to that of the injected spin polarized electrons. In particular, the spin-orbit interaction may affect the zero-conductance resonances in asymmetrically coupled one-dimensional rings. ${ }^{49}$ Also, because of the importance of this interaction, quantum rings have been proposed as electron-spin beam filters. ${ }^{50}$

It is worth noting that, in general, all the above effects require numerical investigation. The advantage of the simple waveguide electron model presented in this work consists in finding simple analytical expressions that enable us to discuss the existence of Fano resonances as well as the effect of the different wire lengths in tailoring these resonances without incorporating a defect (a dot) in one of the two arms of the ring, as it is usually the case in such mesoscopic systems. ${ }^{51}$ Such a model can also give a qualitative good description of the experimental Fano line shapes in onedimensional narrow wires at low temperature. ${ }^{52,53}$ We hope that the effects reported in this paper about the electronic scattering and transport in mesoscopic structures will stimulate further experimental and theoretical interest on similar mesoscopic devices.

\section{ACKNOWLEDGMENTS}

H.A.-W. and E.H.E.B. gratefully acknowledge the hospitality of the Institut d'Electronique, de Microélectronique et de Nanotechnologie (IEMN), UMR CNRS 8520 and UFR de Physique, Université des Sciences et Technologies de Lille. This work was supported by "le Fonds Européen de Développement Régional" (FEDER), INTERREG III FranceWallonie-Flandre (PREMIO) and "le Conseil Régional NordPas de Calais."
*Corresponding author. Electronic address: abdellatif.akjouj@univlille1.fr

${ }^{1}$ See, e. g., S. Datta, Electronic Transport in Mesoscopic Systems (Cambridge University Press, Cambridge, 1995); Proceedings of the 13th International Conference on Electronic Properties of Two-Dimensional Systems (EP2DS) [Physica E (Amsterdam) 6, 1 (2000)].

${ }^{2}$ Y. Cui, Q. Wai, H. Park, and C. M. Liber, Science 293, 1289 (2001).

${ }^{3}$ J. Wang, M. S. Gudiksen, X. Duan, Y. Cui, and C. M. Lieber, Science 293, 1455 (2001).

${ }^{4}$ D. Whang, S. Jin, Y. Wu, and C. M. Lieber, Nano Lett. 3, 1255 (2003).

${ }^{5}$ X. Duan, C. Niu, V. Sahi, J. Chen, J. W. Parce, S. Empedocles, and J. L. Goldman, Nature (London) 425, 274 (2003).

${ }^{6}$ R. Kucharczyk and S. G. Davison, Phys. Rev. B 69, 195402 (2004)

${ }^{7}$ R. Kucharczyk, L. Dobrzynski, B. Djafari-Rouhani, and A. Ak- jouj, Physica E (Amsterdam) 24, 355 (2004).

${ }^{8}$ The Handbook of Microlithography, Micromachining, and Microfabrication, edited by P. Rai-Chaudhry (SPIE, Bellingham, 1997).

${ }^{9}$ U. Fano, Phys. Rev. 124, 1866 (1961); D. Goldhaber-Gordon, H. Shtrikman, D. Mahalu, D. Abush-Magder, U. Meirav, and M. A. Kastner, Nature (London) 391, 156 (1988); J. Göres, D. Goldhaber-Gordon, S. Heemeyer, M. A. Kastner, H. Shtrikman, D. Mahalu, and U. Meirav, Phys. Rev. B 62, 2188 (2000); Y. Ji, M. Heiblum, and H. Shtrikman, Phys. Rev. Lett. 88, 076601 (2002).

${ }^{10}$ K. Kobayashi, H. Aikawa, S. Katsumoto, and Y. Iye, Phys. Rev. Lett. 88, 256806 (2002); J. Kim, J.-R. Kim, Jeong-O. Lee, J. W. Park, H. M. So, N. Kim, K. Kang, K.-H. Yoo, and J.-J. Kim, ibid. 90, 166403 (2003); B. Babic and C. Schönenberger, Phys. Rev. B 70, 195408 (2004).

${ }^{11}$ Y. Gefen, Y. Imry, and M. Y. Azbel, Phys. Rev. Lett. 52, 129 (1984). 
${ }^{12}$ A. Yacoby, M. Heiblum, D. Mahalu, and H. Shtrikman, Phys. Rev. Lett. 74, 4047 (1995); R. Schuster, E. Buks, M. Heiblum, D. Mahalu, V. Umansky, and H. Shtrikman, Nature (London) 385, 417 (1997).

${ }^{13}$ L. P. Levy, G. Dolan, J. Dunsmuir, and H. Bouchiat, Phys. Rev. Lett. 64, 2074 (1990); P. S. Deo and A. M. Jayannavar, Mod. Phys. Lett. B 7, 2074 (1990); D. Mailly, C. Chapelier, and A. Benoit, Phys. Rev. Lett. 70, 2020 (1993).

${ }^{14}$ C. Benjamin and A. M. Jayannavar, Phys. Rev. B 64, 233406 (2001); P. S. Deo and A. M. Jayannavar, ibid. 50, 11629 (1994).

${ }^{15}$ A. A. Clerk, X. Waintal, and P. W. Brouwer, Phys. Rev. Lett. 86, 4636 (2001).

${ }^{16}$ J. F. Song, Y. Ochiai, and J. P. Boid, Appl. Phys. Lett. 82, 4561 (2003).

${ }^{17}$ Y. S. Joe, A. M. Satanin, and G. Klimeck, Phys. Rev. B 72, 115310 (2005).

${ }^{18}$ M. L. Ladron de Guevara, F. Claro, and P. A. Orellana, Phys. Rev. B 67, 195335 (2003); K.-K. Voo and C. S. Chu, ibid. 72, 165307 (2005); G. Hackenbroich and H. A. Weidenmüller, Phys. Rev. Lett. 76, 110 (1996).

${ }^{19}$ J. Wu, B.-L. Gu, H. Chen, W. Duan, and Y. Kawazoe, Phys. Rev. Lett. 80, 1952 (1998); T. Taniguchi and M. Buttiker, Phys. Rev. B 60, 13814 (1999); H. W. Lee, Phys. Rev. Lett. 82, 2358 (1999).

${ }^{20}$ L. Dobrzynski, Surf. Sci. Rep. 11, 139 (1990).

${ }^{21}$ J. O. Vasseur, A. Akjouj, L. Dobrzynski, B. Djafari-Rouhani, and E. H. El Boudouti, Surf. Sci. Rep. 54, 1 (2004).

${ }^{22}$ A. Akjouj, L. Dobrzynski, B. Djafari-Rouhani, J. O. Vasseur, and M. S. Kushwaha, Europhys. Lett. 41, 321 (1998).

${ }^{23}$ H. Al-Wahsh, A. Akjouj, B. Djafari-Rouhani, L. Dobrzynski, A. Mir, N. Fettouhi, M. Tij, and A. Bouzid, Europhys. Lett. 57, 711 (2002).

${ }^{24}$ M. Büttiker and R. Landauer, Phys. Rev. Lett. 49, 1739 (1982); M. Büttiker, Phys. Rev. B 27, 6178 (1983); E. H. Hauge and J. A. Stovneng, Rev. Mod. Phys. 61, 917 (1989); V. Laude and P. Tournois, J. Opt. Soc. Am. B 16, 194 (1999).

${ }^{25}$ M. L. H. Lahlaouti, A. Akjouj, B. Djafari-Rouhani, L. Dobrzynski, M. Hammouchi, E. H. El Boudouti, A. Nougaoui, and B. Kharbouch, Phys. Rev. B 63, 035312 (2001).

${ }^{26}$ H. Barkay, E. Narevicius, and N. Moiseyev, Phys. Rev. B 67, 045322 (2003); I. Rotter and A. F. Sadreev, Phys. Rev. E 71, 046204 (2005).

${ }^{27}$ E. H. El Boudouti, N. Fethouhi, A. Akjouj, B. Djafari-Rouhani, A. Mir, J. O. Vasseur, L. Dobrzynski, and J. Zemmouri, J. Appl. Phys. 95, 1102 (2004).

${ }^{28}$ A. Aharony, O. Entin-Wohlman, B. I. Halperin, and Y. Imry, Phys. Rev. B 66, 115311 (2002).
${ }^{29}$ L. Brillouin, Wave Propagation and Group Velocity (Academic, New York, 1960).

${ }^{30}$ S. G. B. Garret and D. E. McCumber, Phys. Rev. A 1, 305 (1970).

${ }^{31}$ S. Chu and S. Wong, Phys. Rev. Lett. 48, 738 (1982).

${ }^{32}$ L. J. Wang, A. Kusmich, and A. Dogardin, Nature (London) 277, 406 (2000)

${ }^{33}$ J. N. Munday and W. M. Robertson, Appl. Phys. Lett. 81, 2127 (2002).

${ }^{34}$ W. M. Mitchell and R. Y. Chiao, Am. J. Phys. 66, 14 (1998).

${ }^{35}$ B. Macke and B. Segard, Eur. Phys. J. D 23, 125 (2003).

${ }^{36}$ H. G. Winful, Phys. Rev. Lett. 90, 023901 (2003).

${ }^{37}$ B. J. van Wees, H. van Houten, C. W. J. Beenakker, J. G. Williamson, L. P. Kouwenhoven, D. van der Marel, and C. T. Foxon, Phys. Rev. Lett. 60, 848 (1988).

${ }^{38}$ P. Debray, O. E. Raichev, P. Vasilopoulos, M. Rahman, R. Perrin, and W. C. Mitchell, Phys. Rev. B 61, 10950 (2000).

${ }^{39}$ G. Kirczenow, Phys. Rev. B 39, 10452 (1989); L. C. O. Dacal, A. J. Damiao, and E. A. de Andrada e Silva, ibid. 71, 155330 (2005).

${ }^{40}$ H. Wu, D. W. L. Sprung, J. Martorell, and S. Klarsfeld, Phys. Rev. B 44, 6351 (1991).

${ }^{41}$ R. Akis, P. Vasilopoulos, and P. Debray, Phys. Rev. B 52, 2805 (1995).

${ }^{42}$ K. F. Berggren and Z. L. Ji, Phys. Rev. B 43, 4760 (1991).

${ }^{43}$ J. P. Carini, J. T. Londergan, and D. P. Murdock, Phys. Rev. B 55, 9852 (1997); J. P. Carini, J. T. Londergan, D. P. Murdock, D. Trinkle, and C. S. Yung, ibid. 55, 9842 (1997).

${ }^{44}$ Y. Wang, J. Wang, H. Guo, and E. Zaremba, Phys. Rev. B 52, 2738 (1995).

${ }^{45}$ A. C. Johnson, C. M. Marcus, M. P. Hanson, and A. C. Gossard, Phys. Rev. Lett. 93, 106803 (2004).

${ }^{46}$ V. Moldoveanu, M. Tolea, and B. Tanatar, Phys. Rev. B 75, 045309 (2007).

${ }^{47}$ E. Buks, R. Schuster, M. Heiblum, D. Mahalu, V. Umansky, and H. Shtrikman, Phys. Rev. Lett. 77, 4664 (1996).

${ }^{48}$ A. A. Kiselev and K. W. Kim, J. Appl. Phys. 94, 4001 (2003).

${ }^{49}$ U. Aeberhard, K. Wakabayashi, and M. Sigrist, Phys. Rev. B 72, 075328 (2005).

${ }^{50}$ P. Földi, O Kàlman, M. G. Benedict, and F. M. Peeters, Phys. Rev. B 73, 155325 (2006).

${ }^{51}$ P. Vasilopoulos, O. Kàlman, F. M. Peeters, and M. G. Benedict, Phys. Rev. B 75, 035304 (2007).

${ }^{52}$ A. Fuhrer, P. Brusheim, T. Ihn, M. Sigrist, K. Ensslin, W. Wegscheider, and M. Bichler, Phys. Rev. B 73, 205326 (2006).

${ }^{53}$ O. Entin-Wohlman, A. Aharony, Y. Imry, Y. Levinson, and A. Schiller, Phys. Rev. Lett. 88, 166801 (2002). 